\title{
Revisiting the effect of quality of graphics on distance judgments in virtual environments: A comparison of verbal reports and blind walking
}

\author{
Benjamin R. Kunz, Leah Wouters, Daniel Smith, \\ William B. Thompson, ANd SARAH H. CreEM-Regehr \\ University of Utah, Salt Lake City, Utah
}

\begin{abstract}
In immersive virtual environments, judgments of perceived egocentric distance are significantly underestimated, as compared with accurate performance in the real world. Two experiments assessed the influence of graphics quality on two distinct estimates of distance, a visually directed walking task and verbal reports. Experiment 1 demonstrated a similar underestimation of distances walked to previously viewed targets in both low- and high-quality virtual classrooms. In Experiment 2, participants' verbal judgments underestimated target distances in both graphics quality environments but were more accurate in the high-quality environment, consistent with the subjective impression that high-quality environments seem larger. Contrary to previous results, we suggest that quality of graphics does influence judgments of distance, but only for verbal reports. This behavioral dissociation has implications beyond the context of virtual environments and may reflect a differential use of cues and context for verbal reports and visually directed walking.
\end{abstract}

In head-mounted-display-(HMD-)based immersive virtual environments (VEs), observers underestimate absolute egocentric distance. They walk, throw, and verbally respond as if distances are closer than they are intended to be (Durgin, Fox, Lewis, \& Walley, 2002; Loomis \& Knapp, 2003; Mohler, Creem-Regehr, \& Thompson, 2006; Richardson \& Waller, 2007; Sahm, Creem-Regehr, Thompson, \& Willemsen, 2005; Thompson et al., 2004; Willemsen, Gooch, Thompson, \& Creem-Regehr, 2008). Now, numerous research studies have explored many of the possible reasons for this systematic effect on distance judgments. One intuitive factor, and the manipulation in the present study, is the nature of the computer graphics. The cartoon-like nature of some graphics used in VEs may influence perceptual fidelity by affecting cues for absolute distance, such as familiar size. Subjective experience supports this claim, since more realistic, higher-graphicsquality-rendered spaces seem larger than equivalently sized spaces rendered with less realistic textures and objects. In contrast to this anecdotal experience, Thompson et al. (2004) found no significant difference in egocentric distance judgments between three distinctly different qualities of graphics presented in a VE. Thompson et al.'s (2004) study focused on one type of distance estimation, triangulation by walking, in which observers viewed a target and walked indirectly toward that target. The present study builds on this work by asking a question more targeted to perceptual mechanisms, examining whether the effects of quality of graphics are generalizable across two different types of response measures. Given the striking contrast between one's "sense" of the size of the space and the objective measure of visually directed walking, we questioned whether quality of graphics might affect verbal reports of distance more than it does a visually directed walking measure. We conducted two experiments to assess the potential dissociation between visually directed walking and verbal reports of distance in HMD-based VEs as a function of the quality of graphics. Assessing the generalizability of the effects of quality of graphics on distance judgments has both theoretical implications for the unitary nature of perception and action and applied significance for the utility of HMD-based VEs in domains such as training, education, and rehabilitation.

\section{Distance Perception in Virtual Environments}

Simulations used for training, education, and prototype/ model walkthroughs should reliably convey real-world spatial characteristics and constraints in order for knowledge and skills acquired in VEs to transfer seamlessly to the real world. For example, a number of studies have suggested that changes in perceptual-motor calibration induced in VEs can significantly influence subsequent actions performed in the real world (Fox \& Durgin, 2003; Mohler et al., 2006; Mohler et al., 2007). For research purposes, VEs offer an opportunity to manipulate sensory and environmental cues that are less easily manipulated in the real world. Distance perception is particularly important for practical applications utilizing immersive VEs as simulations of real-world scenarios and environments, but practical and experimental use of VE technology is limited by users' nonveridical

B. R. Kunz, benjamin.kunz@psych.utah.edu 
perception of the space of VEs presented in HMDs. This misperception of virtual spaces is apparent in subjective judgments of HMD-based VEs as appearing smaller than they are intended to be and has been systematically demonstrated in a number of experiments assessing judgments of absolute egocentric distance. These experiments have consistently demonstrated that VE users judge the distances to targets as shorter than they are intended to be. Underestimation of distance has been demonstrated through verbal reports of distance (Mohler et al., 2006), blind walking to previously viewed targets (Durgin et al., 2002; Loomis \& Knapp, 2003; Richardson \& Waller, 2007; Willemsen \& Gooch, 2002; Witmer \& Sadowski, 1998), and throwing measures (Sahm et al., 2005) and are unlikely in isolation to be the result of the limited field of view or the mechanics of HMDs (Willemsen, Colton, Creem-Regehr, \& Thompson, 2009), stereo vision conflicts (Willemsen et al., 2008), or optical distortions inherent to HMDs (Kuhl, Thompson, \& Creem-Regehr, 2008).

The hypothesis that the quality of the graphics in HMD-based VEs affects distance judgments was tested by Thompson et al. (2004) in a study involving visually directed walking along an indirect path toward a target. It was predicted that distance judgments might be more accurate with improved image quality, with the rationale that low-quality VE graphics are missing subtle but important cues for size and distance, such as texturing and illumination. Informal observations by Loomis and Knapp (2003) suggested improvements in accuracy, given a real environment viewed via direct camera feeds through the HMD, as compared with a graphical HMD environment; however, Messing and Durgin (2005) found the camera feed HMD performance to be significantly worse than real-world controls. Thompson et al. (2004) used a between-subjects design in which participants viewed the same environment rendered as either wire-frame graphics, low-quality graphics containing simple geometry and low spatial-frequency texture maps, or high-quality/photorealistic images made using panoramic photographs of the actual environment. Despite subjectively reported experience of greater realism, there were no significant differences in performance on the walking task as a function of graphics quality. In short, the participants judged the distances to be shorter than intended regardless of the quality of the graphics used to present target and environmental information. This surprising result does not invalidate the experience of improved perceived realism and a subjectively larger space with better graphics but does suggest that more empirical work is needed to determine how best to measure this phenomenological experience and to explain potential differences between response measures.

Our goal was to determine whether the phenomenological experience assessed informally would be more formally revealed in a measure involving verbal reports of distance and whether this would dissociate behaviorally from a visually directed walking task. The prediction that higher quality of graphics will improve verbal reports has implications beyond the practical results for VE underestimation in itself. More importantly, in the context of theories of space perception, we can use findings of a be- havioral dissociation as a function of graphics manipulation to examine several hypotheses relating to why different response measures may result in differential distance estimates. Whereas the aim of Thompson et al. (2004) was to find an explanation in the quality of graphics that could be used to improve performance in VEs, our primary goal was to test whether the effects of the quality of graphics might inform us about the mechanisms underlying distance estimations as a function of the response required.

\section{Measures of Distance Perception}

Assessing absolute distance perception beyond reachable distances is difficult because of the necessity of inferring perceptual experience from behavioral response (Loomis \& Philbeck, 2008). Two broad categories of measures have been used: conscious report/magnitude estimation of the distance to a target versus a visually directed action toward a target location. Magnitude estimations include verbally indicated judgments of the distance, as well as other traditional psychophysical measures such as extentmatching tasks. Action-based judgments have involved throwing, walking, pointing, or some combination of these tasks and require that an observer view a target location and then perform the action without visual feedback. The accuracy of the action is thought to reflect the accuracy of the perception of distance. For assessing space perception in VEs, action-based tasks typically have greater ecological validity, since they are generally related to actions that are performed within real and virtual environments (such as walking through or acting on spaces). There are different theoretical viewpoints about how closely related action- and nonaction-based measures of distance are with respect to internal representations of distance. Although there are well-known theories in perception that argue for dissociable representations for conscious perception and visually guided actions (Bridgeman, Lewis, Heit, \& Nagle, 1979; Milner \& Goodale, 1995), there are limitations to this model, particularly as it applies to blind walking as a response measure. There is also behavioral evidence in support of a common representation informing multiple action- and nonaction-based measures of distance (see Loomis \& Philbeck, 2008, for a review).

In general, verbal reports of distance in the real world tend to be more variable and less accurate than actionbased measures of distance (Andre \& Rogers, 2006; Kelly, Loomis, \& Beall, 2004; Loomis \& Philbeck, 2008). In contrast, blind walking in particular seems to be a consistent, reliable measure of distance perception and has arguably received the most recent use as a measure of distance perception. A number of real-world studies converge on the finding that participants can walk accurately to previously viewed targets up to $20 \mathrm{~m}$ away (Andre \& Rogers, 2006; Loomis, Da Silva, Fujita, \& Fukusima, 1992; Loomis \& Philbeck, 2008; Rieser, Ashmead, Talor, \& Youngquist, 1990). In the most common variant of this task, participants view a target in a real-world environment; the participant is then blindfolded and instructed to walk to the location of the target. The distance walked is used as the critical measure of judged distance. Triangulation or indirect walking tasks have also been employed to 
eliminate the potential for planning a motor or cognitive response during the viewing stage of blind walking. In these tasks, judged distance to a target is derived from the participant's original viewpoint and the intersection of the viewing line of sight and the final indicated direction. In an example of this type of task, participants view a target and then walk without vision at an oblique angle relative to the target. Upon a cue from the experimenter, the blindfolded participant stops, turns, and either points to the perceived location of the previously viewed target or turns and walks toward the target location. Although somewhat more variable than direct blind walking, performance in triangulated walking tasks is also quite accurate (Fukusima, Loomis, \& Da Silva, 1997), although there is some variability across different types of triangulated walking and pointing tasks (Philbeck, Loomis, \& Beall, 1997).

Despite some indications of potential differences between verbal reports of distance and blind-walking performance, only a few studies have directly compared these two measures. A recent review by Loomis and Philbeck (2008) suggested that real-world performance for the two tasks reflects a unitary perception of distance. The authors cited several experiments in which differences between distance walked and distance reported persisted; however, Philbeck and Loomis (1997) have found that manipulations of available visual cues affect both types of measures similarly, suggesting that both measures are informed by the same representation. In contrast, Andre and Rogers (2006) compared verbal reports and blind-walking judgments in the real world and reported significant differences in performance of these two tasks. Blind walking was more accurate than verbal reports in a baseline comparison; verbal reports of distances viewed in an outdoor environment were underestimated more than those viewed in an indoor, carpentered environment, whereas blindwalking judgments were unaffected by environment; and prism goggle viewing affected blind-walking performance more than it did verbal reports of distance. In sum, there is controversy regarding the existence and nature of any differences between nonaction-based and action-based measures of distance perception.

In the context of the present work, a comparison of the two types of response measures is important for two reasons. First, a dissociation of response as a function of quality of graphics could provide insights into theoretical questions associated with whether or not different measures rely on the same or different internal representations and the extent to which the use of a response measure may influence the way the task is solved. Second, assessing similarities and differences among measures of distance perception in HMD-based VEs could have direct implications for generalization across different measures that may be used both to evaluate and to improve the potential use of HMD-based VEs for applications.

\section{Overview of the Experiments}

We conducted two experiments to examine the effects of quality of graphics on estimations of absolute egocentric distance. In the first experiment, we assessed direct blind walking to previously viewed targets in a VE. We used two quality-of-graphics conditions: a low-quality graphics model with simple geometry and low-spatial-frequency tiled textures and a high-quality graphics model with photorealistic textures applied to more complex and realistic computer-generated geometry. This experiment served to test whether the results in Thompson et al. (2004) could be replicated with a different action-based walking measure. Whereas Thompson et al. (2004) utilized a triangulated walking measure, we employed a direct blind-walking measure that typically results in less variable performance and that has been more extensively used in VE work on distance perception. In Experiment 2, we utilized the same VE with the two quality-of-graphics conditions but tested the effects on verbal reports of distance. The results indicated that blind walking was not influenced by the quality of the graphics, whereas verbal reports of distance were significantly improved with the use of high-quality graphics. These findings highlight the importance of utilizing multiple measures to assess spatial perception and lead to several possible interpretations of the nature of behavioral distance estimations.

\section{EXPERIMENT 1}

\section{Method}

Participants. A total of 26 participants were randomly assigned to either a high-quality $(n=13)$ or low-quality $(n=13)$ graphics condition. Undergraduate participants were compensated with credit toward fulfilling a course requirement; participants from the university community were compensated with $\$ 10$ for their participation. The participants were screened for stereo vision and visual acuity. Each participant was run through the experiment individually over the course of approximately $1 \mathrm{~h}$.

Materials. An immersive VE was presented to the participants, using an NVIS nVisor SX HMD with a progressive-scan 1,280 $\times$ 1,024 pixel resolution, approximately $42^{\circ}$ horizontal field of view, and $100 \%$ stereo overlap between the two eyes. Participant head movements were tracked using an InterSense IS600 Mark 2 tracker, which employs acoustical and inertial signals to provide low-latency, highly accurate position tracking. HMD optical pincushion distortion was corrected using a graphics card shader program, without introducing additional latency (see Kuhl et al., 2008, for an explanation of HMD calibration and correction).

The VEs consisted of a virtual classroom measuring $13.3 \times 10.1 \mathrm{~m}$, modeled after a real-world classroom (see Figure 1). In the low-quality condition, the participants viewed the virtual classroom with reduced geometry and very little visual detail (low-spatial-frequency generic textures applied to the walls, ceiling, and floor). Virtual targets were randomly selected from a group of six targets of different size, color, and shape; virtual targets were also rendered without detailed shadow or texture information in the low-quality condition. The high-quality condition consisted of the same classroom with photorealistic surface textures applied to computer-generated geometry, including objects of familiar size (e.g., desk, wall clock, etc.). Virtual targets were the same as those used in the low-quality condition, but with photorealistic textures and realistic shadows applied.

Noise-canceling headphones were used to reduce sound localization as a cue to position tracking, and a wireless microphone was used to avoid acoustic localization of the experimenter.

Procedure. After providing informed consent, the participants were tested for visual acuity. Participants unable to read an eye chart at 20/30 or better or who demonstrated lack of stereo fusion were excluded from the analysis. After reading written instructions for the task, the participants were outfitted with noise-canceling headphones and a blindfold and were informed about the general nature of the task to be completed. The participants then practiced blind 

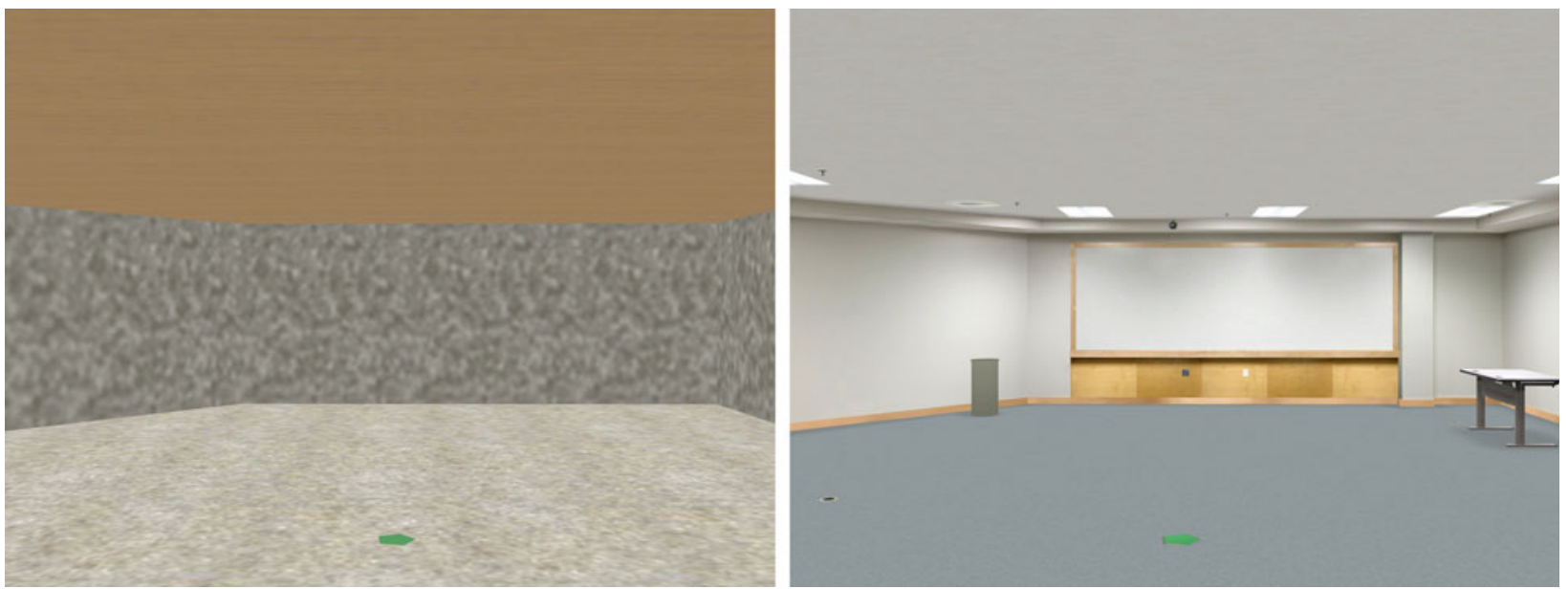

Figure 1. Images of the low-quality (left) and high-quality (right) classroom graphical models.

walking in a hallway, first being led by an experimenter by the shoulders and then walking independently with an experimenter following behind and providing verbal indications of when to turn and stop. After approximately 5 min of blind walking, or until the participants demonstrated and professed comfort with blind walking, the experimenter verbally described and demonstrated the experimental task.

The participants were instructed to view a target at a given distance and to form a "good image" of the target and the surroundings. A "good image" was defined as one that, once obtained, would allow the participants to visualize the environment and target after their eyes were closed. Once the participants obtained this image, they were instructed to close their eyes and walk to the target location. The participants informally practiced the tasks twice in the real world (without performance feedback), reinforcing the written and verbal instructions for the participants and allowing the experimenter to ensure proper understanding of the task. After a brief practice in the hallway adjacent to the VE lab, the participants were blindfolded and led into the lab.

Once inside the lab, the participants were outfitted with the HMD without viewing the physical lab space. While wearing the HMD, depending upon condition, the participants viewed a virtual target consisting of a colored shape on the ground plane at a distance of 3, 4.5, or $6 \mathrm{~m}$ (each repeated three times) in either the high-quality or the low-quality VE. Two dummy trials (targets at distances of 3.75 and $5.25 \mathrm{~m}$ ) were randomly interspersed to reduce the use of memory strategies for the three critical target distances; the data from these trials were not analyzed. On each trial, the participants were allowed to view the target as long as necessary and were prompted to move their heads by turning (without moving from their starting location or moving side-to-side) to view the room. Once the participants were satisfied with the "good image" of the target and environment, they closed their eyes (the HMD screen was blanked) and walked to the target location. Distance walked was recorded using the InterSense tracking system. After reaching the judged target location, the participants were guided back to the starting point via a circuitous route through the lab. Although the physical starting point remained the same, the participants viewed one of three different starting points in the virtual classroom (randomly selected from one of three offsets along the participants' heading direction). As with the dummy trials, the varied starting points were intended to reduce the memorization of target distances and associations between targets and specific locations within the classroom. After 2 practice trials (targets presented at $3.75 \mathrm{~m}$ and $5.25 \mathrm{~m}$ ) participants completed 11 trials (including the two dummy trials). For each participant, distances walked to the targets at $3,4.5$, and $6 \mathrm{~m}$ (each repeated three times) were averaged for subsequent analysis. No feedback regarding performance was given until after the experimental session.

\section{Results and Discussion}

Quality of graphics had no significant effect on distances walked, and both graphics conditions showed substantial underestimation of distance. A 2 (quality of graphics) $\times 3$ (distance to target) repeated measures ANOVA performed on distance walked, with quality as a betweensubjects variable and distance as a within-subjects variable revealed a significant main effect of distance to target $\left[F(2,48)=421.47, p=.001, \eta_{\mathrm{p}}^{2}=.946\right]$ but no main effect of quality of graphics $[F(1,24)=1.629, p=.214$, $\left.\eta_{\mathrm{p}}^{2}=.064\right]$ and no interaction between quality of graphics and distance $\left[F(2,48)=2.285, p=.113, \eta_{\mathrm{p}}^{2}=.087\right]$. Planned contrasts revealed a significant difference between distances walked to 3 - and 4.5-m targets $[F(1,24)=$ $\left.405.15, p<.001, \eta_{\mathrm{p}}^{2}=.944\right]$, as well as to 4.5 - and 6-m targets $\left[F(1,24)=269.41, p<.001, \eta_{\mathrm{p}}^{2}=.918\right]$. Mean estimations of distances in Experiment 1 are presented in Figure 2. Averaged across distance, the mean distance

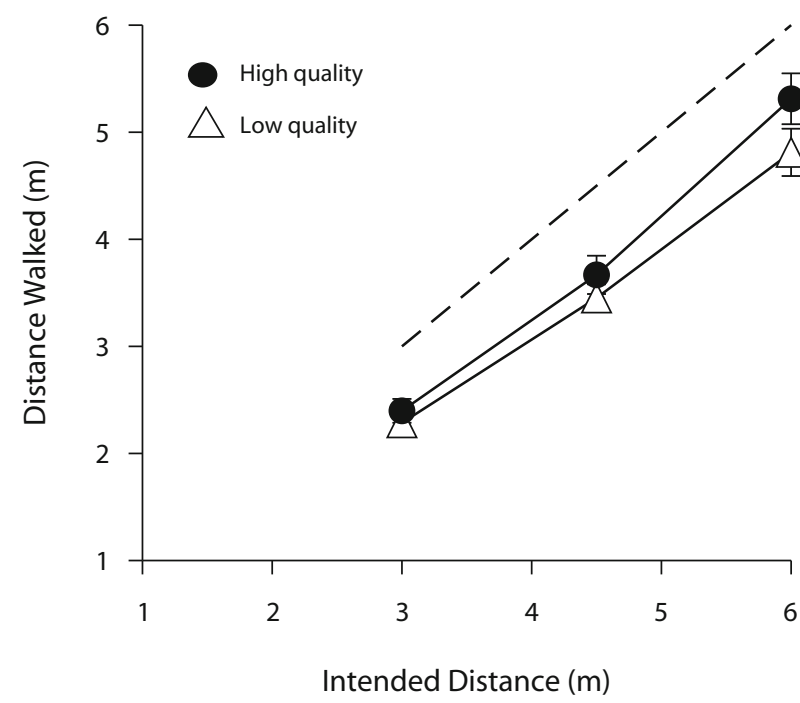

Figure 2. Mean blind-walking distances for high- and lowquality virtual rooms. Error bars represent one standard error above and below the mean. 
Table 1

Variable Error by Distance for Experiment 1

\begin{tabular}{cccc}
\hline & \multicolumn{3}{c}{ Distance $(\mathrm{m})$} \\
\cline { 2 - 4 } Quality & 3 & 4.5 & 6 \\
\hline Low & 0.207 & 0.405 & 0.458 \\
High & 0.182 & 0.360 & 0.463 \\
\hline
\end{tabular}

walked in the high-quality room was $83.33 \%$ of the actual distance to the target, and the mean distance walked in the low-quality room was $77.71 \%$ of the actual distance to the target.

Within-subjects variability was computed for each target distance as the standard deviation of the mean across three trials at each distance for each participant and was then analyzed as a measure of variable error. A 2 (quality of graphics) $\times 3$ (distance to target) repeated measures ANOVA performed on variable error revealed a significant main effect of distance to target $[F(2,48)=17.36$, $\left.p=.001, \eta_{\mathrm{p}}^{2}=.420\right]$ but no effect of quality of graphics $\left[F(1,24)=0.619, p=.439, \eta_{\mathrm{p}}^{2}=.025\right]$ and no interaction between distance to target and quality of graphics $\left[F(2,48)=0.027, p=.973, \eta_{\mathrm{p}}^{2}=.001\right]$. Planned contrasts revealed a significant difference between 3 - and 4.5 -m targets $\left[F(1,24)=34.889, p<.001, \eta_{\mathrm{p}}^{2}=.592\right]$ but no significant difference between 4.5 - and $6-\mathrm{m}$ targets $\left[F(1,24)=1.451, p=.240, \eta_{\mathrm{p}}^{2}=.057\right]$. Variable errors for Experiment 1 are presented in Table 1. Consistent with other studies on blind walking, there was an increase in variable error with distance, but this did not vary as a function of the quality of graphics.

\section{EXPERIMENT 2}

The results of Experiment 1 demonstrate that quality of graphics had little effect on a direct blind-walking measure of egocentric distance, similar to the findings in Thompson et al. (2004), in which a different visually directed walking task, a different environment, and different levels of quality were used. The results in the present experiment for both levels of quality were consistent with previous work in our laboratory showing about $80 \%$ accuracy in direct blind walking to target locations. These results are important in that they serve as a replication of an earlier study in which different methods were used and also demonstrate a distinction in a clearly different subjective sense of realism between the two levels of graphic quality but little impact on a perceptual judgment. Experiment 2 tested this distinction further by introducing verbal report as a response measure, using otherwise the same environments and methodology.

\section{Method}

Participants. A total of 28 participants from the university community participated in Experiment 2. As in Experiment 1, the participants were randomly assigned to either a high-quality $(n=14)$ or a low-quality $(n=14)$ graphics condition. Only the participants who professed greater familiarity with the U.S. customary system of measurements than with metric standards were included in the final analysis. The participants were compensated as described in Experiment 1.
Materials. The same HMD, tracking system, noise canceling equipment, and VEs as those previously described were used in Experiment 2. Because the participants in Experiment 2 were instructed to verbally report judged target distance, they were familiarized with a common metric. The participants in Experiment 2 viewed a wooden dowel cut to a 1-yard length. Each foot along the wooden dowel was clearly marked, but no other markings appeared along the dowel.

Procedure. The procedure for Experiment 2 closely followed that in Experiment 1, except that the participants did not walk to previously viewed targets but, instead, called out the estimated distance to the target. Following initial procedures described in Experiment 1 (written instructions, vision acuity tests, practice blind walking), the participants were familiarized with a standard metric for making verbal judgments of distance. Prior to two informal practice trials in the real world, the participants viewed the 1-yard metric (the wooden dowel described above) held horizontally in front of them at a distance of approximately $1 \mathrm{~m}$ and were informed about the dowel's length and the 1 - $\mathrm{ft}$ markings. During practice trials and experimental trials, the dowel was not visible.

In two informal practice trials in the real-world hallway adjacent to the VE lab, the participants were instructed to view a target at a given distance and to report the judged distance to the target. The participants were instructed to be as precise as possible and to use whole units and fractions (e.g., $5 \frac{3 / 4}{\mathrm{ft}}$ ) to judge the distance. No feedback was given for these practice trials. Following this practice, the participants were blindfolded and led into the lab.

Once outfitted with the HMD, the participants viewed either the high-quality or the low-quality environment (depending on condition). The environments, targets, and distances used were all identical to those previously described in Experiment 1. On each trial, the participants were allowed to view the target as long as necessary and, when ready, to call out the distance to the target, using feet and fractions of feet. The verbal report was given with eyes open to reduce any potential memory-related influences on verbal judgments of distance. The eyes-open verbal reports procedure is consistent with recent distance judgment experiments that employed both blind walking and verbal reports (e.g., Andre \& Rogers, 2006; Philbeck $\&$ Loomis, 1997). Immediately after reporting the distance, the participants were instructed to close their eyes, the HMD display was blanked, and the participants were then guided by the experimenter through the experiment space via a circuitous route to the starting position of the next trial. The presentation of experimental trials was the same as that in Experiment 1. After 2 practice trials (targets presented at 3.75 and $5.25 \mathrm{~m}$ ), the participants completed 11 randomized trials (including the 2 dummy trials at 3.75 and $5.25 \mathrm{~m}$ ).

For each participant, the verbally reported distances for the targets presented at 3, 4.5, and $6 \mathrm{~m}$ (each repeated three times) were averaged for subsequent analysis. No feedback regarding performance was given until after the experimental session.

\section{Results and Discussion}

In contrast to Experiment 1, the quality of the graphics of the environment had an effect on verbal judgments of egocentric distance. Estimates were greater for the high- than for the low-quality environment. A 2 (quality of graphics) $\times 3$ (distance) repeated measures ANOVA revealed a significant main effect of distance $[F(2,52)=$ $\left.305.20, p<.001, \eta_{\mathrm{p}}^{2}=.921\right]$, as well as a main effect of quality of graphics $\left[F(1,26)=6.458, p=.017, \eta_{\mathrm{p}}^{2}=\right.$ $.199]$, but no interaction between quality of graphics and distance $\left[F(2,52)=1.846, p=.168, \eta_{\mathrm{p}}^{2}=.066\right]$. Planned contrasts revealed a significant difference between distances verbally reported for 3 - and $4.5-\mathrm{m}$ targets $\left[F(1,26)=157.94, p<.001, \eta_{\mathrm{p}}^{2}=.859\right]$, as well as for 4.5 - and 6-m targets $\left[F(1,26)=338.45, p<.000, \eta_{\mathrm{p}}^{2}=\right.$ $.929]$. Mean estimations of distances for Experiment 2 are 


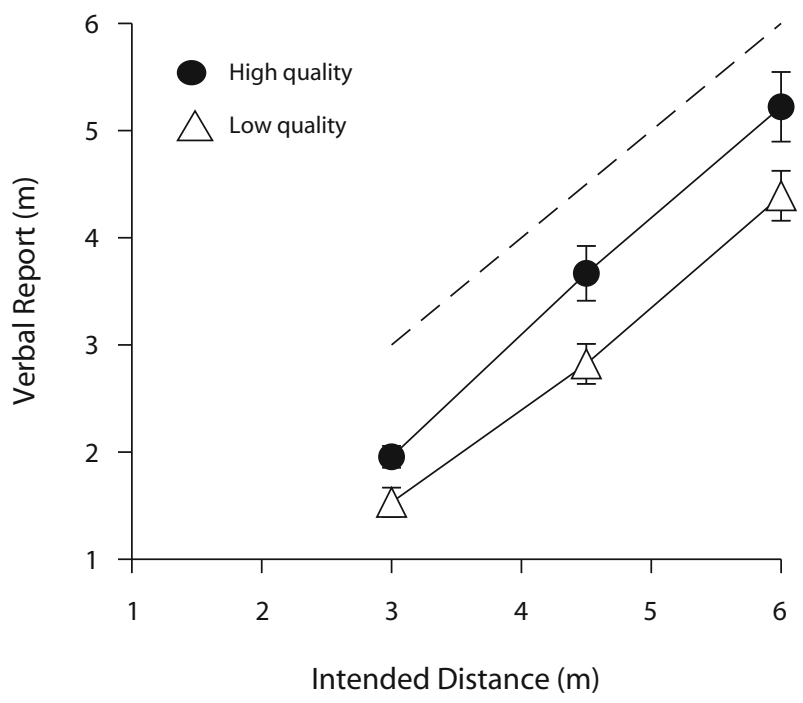

Figure 3. Mean distances verbally reported by participants in high- and low-quality virtual rooms. Error bars represent one standard error above and below the mean.

presented in Figure 3. Averaged across distance, the mean verbal estimate in the high-quality room was $77.91 \%$ of the actual distance to the target, and the mean verbal estimate in the low-quality room was $62.32 \%$ of the actual distance to the target.

Variable error for Experiment 2 was computed in the same manner as in Experiment 1. A 2 (quality of graphics) $\times 3$ (distance to target) repeated measures ANOVA using variable error as the dependent variable revealed a significant main effect of distance to target $[F(2,52)=$ $\left.36.508, p<.001, \eta_{\mathrm{p}}^{2}=.584\right]$ but no effect of quality of graphics $\left[F(1,26)=0.187, p=.669, \eta_{\mathrm{p}}^{2}=.007\right]$ and no interaction between distance to target and quality of graphics $\left[F(2,52)=0.021, p=.980, \eta_{\mathrm{p}}^{2}=.001\right]$. Planned contrasts revealed a significant difference between variable errors at 3- and 4.5-m targets $\left[F(1,26)=10.472, p=.003, \eta_{\mathrm{p}}^{2}=\right.$ .287] and between those at 4.5 - and 6-m targets $[F(1,26)=$ $\left.27.395, p<.001, \eta_{\mathrm{p}}^{2}=.513\right]$. Variable errors for Experiment 2 are presented in Table 2. As in Experiment 1, the observed pattern of increase in variable error is similar for both the low- and high-quality virtual rooms.

\section{Between-Experiments Analysis}

A 2 (experiment/response measure) $\times 2$ (quality of graphics) $\times 3$ (distance) mixed repeated measures ANOVA was conducted in order to further compare the effects of quality of graphics on the two response measures and to compare the two response measures themselves. There was a significant main effect of distance $[F(2,100)=676.22$,

Table 2

Variable Error by Distance for Experiment 2

\begin{tabular}{cccc}
\hline & \multicolumn{3}{c}{ Distance $(\mathrm{m})$} \\
\cline { 2 - 4 } Quality & 3 & 4.5 & 6 \\
\hline Low & 0.203 & 0.339 & 0.696 \\
High & 0.221 & 0.380 & 0.719 \\
\hline
\end{tabular}

$p<.01, \eta_{\mathrm{p}}^{2}=.93$ ], since distance walked increased with target distance. There was a significant effect of experiment/ response measure $\left[F(1,50)=4.85, p<.05, \eta_{\mathrm{p}}^{2}=.088\right]$, with greater average distance estimations in Experiment 1 (blind walking; $M=3.65$ ) than in Experiment 2 (verbal reports; $M=3.26$ ). The overall effect of quality of graphics was also significant, with greater average distance estimations in the high-quality $(M=3.70)$ than in the low-quality $(M=3.20)$ condition. There was, however, no significant experiment/response measure $\times$ quality of graphics interaction $(p=.236)$, despite the finding that additional planned $t$ tests revealed a significant difference in means between blind walking $(M=3.52)$ and verbal reports $(M=$ $2.91)$ in the low-quality condition $[t(25)=2.66, p<.02$, Cohen's $d=1.06]$, but not in the high-quality condition $[t(25)=0.24, p=.52$, Cohen's $d=.26](M=3.79$ and 3.62 , respectively). The lack of a significant interaction is not surprising, due to the lack of a crossover interaction effect. In essence, the between-experiments analysis is consistent with the individual experiment analyses, showing the high-/low-quality blind-walking estimates and the high-quality verbal reports to be similar to each other, as compared with the shorter distance estimates (greater underestimation) seen in the low-quality verbal reports.

\section{GENERAL DISCUSSION}

Together, Experiments 1 and 2 indicate a dissociation between two response measures as a function of the quality of the graphics presented within an HMD VE. Whereas Experiment 1 used blind walking and replicated the previous work by Thompson et al. (2004), showing little effect of the graphics manipulation, Experiment 2 tested verbal reports and showed significant improvement with high versus low graphics quality. Even a quick look at the differences in the images presented in Figure 1 raises the question, why don't we see an effect of graphics quality on blind walking? Although the two models are of the same classroom space, displayed at the same geometric scale, the environmental cues and sense of realism are strikingly different and might be expected to influence all judgments of absolute space.

There are several potential explanations for the observed behavioral dissociations that are important to consider in the framework of models of distance perception. Broadly, we may think of the behavioral distinctions as reflecting either (1) different internal representations of space or (2) a single representation that has been transformed at the time of judgment. One view supporting different representations follows the two-visual-systems hypothesis, suggesting that there are separable visual pathways for perceptual awareness and action that process visual information differently and lead to different behavioral outcomes. This account would suggest that verbal reports reflect an awareness of space that walking-based judgments do not. An alternative account, which also supports different representations, emphasizes task specificity, suggesting that the nature of the response measure may lead the visual system to select different information for distance in constructing a perceptual representation. Following this 
account, quality of graphics influences some visual cues more than others and may, therefore, influence perceptual representations and subsequent behavioral responses in a different way. A third explanation follows the singlerepresentation view, suggesting that the influence of quality of graphics on verbal reports occurs in a judgment process, rather than at the level of internal representation of distance. Below, we will further discuss these three different explanations for the observed behavioral dissociation in the framework of models of distance perception, as well as related research on the effects of environmental context and implications for the applied use of VEs.

\section{Different Representations for Two Visual Systems}

One account of our behavioral results is the model of separable functionally and anatomically defined visual systems, known as the two visual systems, or the dorsal/ ventral processing streams distinction. This model posits that the dorsal or parietal cortex system supports visually guided actions independently from the ventral or inferior temporal cortex system responsible for awareness of objects and space (Milner \& Goodale, 1995, 2008). Interpretations of behavioral dissociations within this model are based on the logic that manipulating one variable and finding two different patterns of results suggests a dissociation of processes. Many empirical studies have tested the theory that representations reflecting awareness of object and spatial attributes are distinct from those used in the context of visually guided action. For example, although a classic visual illusion display such as the Ebbinghaus/ Titchener circles illusion may evoke a size perception bias in phenomenal perception, it has been argued that visually guided grasping of the display remains accurate with respect to the physical size of the object (Aglioti, DeSouza, \& Goodale, 1995; Carey, 2001; but see Franz, 2001, for an alternative account).

Although our results could suggest that the representation informing awareness of distance expressed through verbal reports is fundamentally different from the representation of distance reflected through action, we do not intend to make a strong claim about the fit of our data to the distinction between awareness and action. Behaviorally, there is some evidence against separate representations for awareness and action in distance judgment tasks, demonstrating a tight coupling between visually directed walking and other nonaction measures of perceived distance (Philbeck \& Loomis, 1997) or shape/ size (Hutchison \& Loomis, 2006; Ooi, Wu, \& He, 2006; Wu, Ooi, \& He, 2004). These studies have taken the approach of manipulating cues for distance and then testing whether there are coupled effects on distance and size judgments. The logic underlying this approach is that if there is a common internal representation of distance informing multiple measures, the cue manipulation should affect multiple distance judgments and size judgments, related by size-distance invariance, in a similar way. For example, $\mathrm{Wu}$, Ooi, and He varied the viewing of targets through different-sized apertures and found a coupling between measures of perceived location as assessed by blind walking/gesturing and estimations of size involving the perceived aspect ratio of an L-shaped figure.

Furthermore, it is important to consider closely whether blind walking fits into what is defined as dorsal stream function as neatly as other actions identified with posterior parietal cortex processing, such as grasping. Different from visually guided arm movements, the blind-walking task involves spatial memory, operates over a much longer time frame (several seconds vs. milliseconds), and extends beyond the immediate vicinity of the body (defined by Cutting \& Vishton [1995] as action space vs. personal space). In addition, some neuropsychological findings have suggested that patients with posterior parietal cortex damage can blind walk to targets with performance comparable to that of controls and that it is patients with damage to the medial temporal lobe who have difficulties with these types of spatial updating tasks (Philbeck, Behrmann, Black, \& Ebert, 2000; Philbeck, Behrmann, Levy, Potolicchio, \& Caputy, 2004). However, some recent functional neuroimaging research also has suggested a role for the medial posterior parietal cortex in related spatial updating tasks (Wolbers, Hegarty, Büchel, \& Loomis, 2008). It is possible that future directions in functional neuroimaging approaches will be able to contribute to an understanding of whether different response measures are supported by functionally and neuroanatomically defined perceptual systems. At the same time, further analyses on a behavioral level involving manipulations of attention and visual content of the space, along with multiple response measures, will also likely contribute to an understanding of the present results.

\section{Different Task-Specific \\ Perceptual Representations}

A second account of the present behavioral dissociation proposes different internal representations, as in the first account, but these differences are attributed to taskspecific selection of visual information, rather than to the processing of two separate systems differentiated by conscious experience. Different perceptual representations may be constructed as a function of the response required, and these may be affected differently by the quality-ofgraphics manipulation. Some cues may be used in a similar way across measures, and others may be attended to, or perhaps weighted differently, when distances are reported, as compared with walking them. A related theory about how the visual system uses near ground surface information for intermediate egocentric distance judgments suggests that representations may vary depending on attentional selection of the information $(\mathrm{He}, \mathrm{Wu}$, Ooi, Yarbrough, \& Wu, 2004; Sinai, Ooi, \& He, 1998). For example, when an object was placed between the viewer and the target in an outdoor scene, disrupting available continuous ground surface information, multiple different tasks, including matching, blind throwing, and direct blind walking, showed underestimation of distance (He et al., 2004). However, when the task was indirect walking to the target that bypassed the occluding object, the visual system could select continuous ground surface information, and distances were indicated to be farther. 
In the present study, although some characteristics of the ground surface, such as texture gradients, did change with quality of graphics, one cue that remained constant was angular declination, the angle between an observer's eye level and a visual target. Angular declination has been shown to be a strong cue for absolute distance when scaled by eye height (Ooi, Wu, \& He, 2001; Sedgwick, 1986) and may be used predominantly to construct the internal representation of target location used when blind walking. In contrast, differences in graphics quality affect texture and illumination cues, as well as the presence/absence of other objects, which could serve as familiar size cues to distance. Verbal reports may involve additional attention to these types of context-related cues, leading to a differential influence of context on the perceptual representation of distance for the verbal judgments.

Andre and Rogers (2006) used a related argument to explain distinctions in the effects of environment type and prisms on verbal reports and blind walking in a realworld distance perception study, although they framed it as a distinction between two visual systems. In one experiment, they found that the manipulation of whether the environment was an outdoor athletic training field versus an indoor gymnasium had an effect on verbal reports, but not on blind walking. Whereas blind-walking indications of distance in both environments were quite accurate and not different from each other, verbal reports overall were underestimated, with significantly shorter estimations in the outdoor than in the indoor environment. They argued that verbal reports are more likely to draw on familiar objects for metric scaling, which are more abundant in indoor than in outdoor space. In contrast, use of perspective-based cues scaled by eye height would not likely be affected by more or less of the clutter of objects found in indoor versus outdoor scenes. In a second experiment, Andre and Rogers demonstrated the reverse dissociation. The manipulation of base-down prism goggles led to farther distance estimates through blind walking, but not in verbal reports. It is somewhat difficult to interpret this effect, however, in that the prism effect on blind walking worked only for base-down prisms and not for base-up ones, inconsistent with the results of prior studies (Ooi et al., 2001; Thompson, Dilda, \& Creem-Regehr, 2007). Furthermore, the verbal reports showed a similar trend, which may have been masked by greater variability.

There are a few other recent studies that have suggested effects of environmental context on judgments of space, and these have used primarily verbal reports or matching procedures. Lappin, Shelton, and Rieser (2006) tested judgments of egocentric distance in three real-world naturalistic settings (lobby, hall, open lawn), using a bisection task. Using the method of adjustment, an observer verbally indicated where a person should stand to be at the apparent midpoint of an interval on the ground. They found an antiforeshortening effect for the lobby and the hall, but not for the outdoor lawn, meaning that the midpoint set was overestimated relative to the actual midpoint of the egocentric interval, a finding in contrast to more standard visual space foreshortening effects in which frontal judgments are compressed (Gilinsky, 1951; Loomis et al.,
1992). Witt, Stefanucci, Riener, and Proffitt (2007) also demonstrated environmental context effects on distance judgments associated with a subtle context change in the distance to the endpoint of otherwise similar environments. For both a hallway and an outdoor field, they found that distances to targets that were near a bounded end of the environment were estimated to be farther than those made to targets at the same distance at the unbounded end. The judgment involved a matching task in which the observer viewed a target and then turned in the opposite direction and verbally instructed an experimenter to move closer to or farther from the observer in order to match the distance from the observer to the target. Notably, the variable of the bounded/unbounded environment did not influence direct blind walking to the target location but did influence blind walking when it was performed in the context of a matching judgment (e.g., "walk the perceived distance to the target in the opposite direction"). One interpretation of this difference is that blind walking directly to a target relies on the representation and updating of a spatial location, which is different from the representation used in matching judgments of extent.

It is also important to consider that the present manipulation of graphics quality changed the environmental context in several ways. At the same time, the level of photorealism, geometric complexity, and the presence and familiarity of additional objects in the scene varied. As a result, it is unknown whether image qualities, such as texture and illumination, or object qualities, such as familiar size, led to the present effects on verbal reports. What creates a sense of environmental context is a large question in itself that applies to both the present work and the environmental effects described in the studies above and should be a focus of future work. Although our present results do not define the specific information that may lead to context effects informing the perceptual representation for verbal report judgments, contributing factors could be differences in textures and illumination, the presence of additional surfaces, the presence of familiar-sized objects, and the extent to which object clutter fills a space.

\section{One Perceptual Representation but Different Perceptual Judgments}

A third alternative hypothesis is that the quality of graphics has a differential effect on judgments of distance that may reflect changes in the phenomenology of realism but not the phenomenology of distance perception. This explanation attributes the change in verbal report not to a change in perceptual representation or perceptual awareness of scale, but rather, to a postperceptual judgment process (Hutchison \& Loomis, 2006; Loomis \& Philbeck, 2008). This judgment process itself could be influenced by the perceived realism of the environment.

Support for at least some contribution of cognitive influences on perceptual distance judgments can be seen in the results in Mohler et al. (2006) and Richardson and Waller (2005), in which feedback was provided within an HMD VE with the aim of improving distance estimations. Richardson and Waller (2005) provided participants with explicit feedback about distances walked in a VE with a 
visual display that indicated their accuracy in walking to a target location. In one experiment, the feedback was given for direct blind walking to targets, and postfeedback performance was tested in both direct blind walking and an additional indirect-walking-to-target task. If the feedback had changed a perceptual representation, we would expect a generalized effect of improvement on both measures of distance. However, only the direct blind-walking judgments changed. This finding suggests that a higher level cognitive process such as a rule (e.g., "walk farther than it looks") was used to correct the task-specific judgment. Related studies using several other variations of feedback within the VE have argued that distance judgments in a VE can be changed as a result of multiple processes, including both perceptual-motor recalibration and cognitive correction (Mohler et al., 2006; Richardson \& Waller, 2007; Waller \& Richardson, 2008). Although there is considerable debate as to when changes in perceptual estimates reflect a change in perceptual representation versus a change in a judgment process, the judgment process explanation for the present results cannot be excluded.

\section{Implications}

A final consideration is the practical implications of our present findings. The use of different response measures led to different conclusions about a factor's contribution to the accuracy of distance judgments. The use of VEs for applications that rely on accurate spatial performance may be limited by the bias seen in distance judgments. Our laboratory has made a number of recent attempts to address the problem by investigating the potential differences between HMD-based VEs and the real world (Creem-Regehr, Willemsen, Gooch, \& Thompson, 2005), manipulating the graphical displays (Kuhl et al., 2008) and providing feedback to observers while they are in the VE (Mohler et al., 2006). The present work suggests that for a given approach, it is useful to assess performance in multiple ways. It is important not to assume that all response measures lead to the same results in HMD-based VEs. Testing the generalizability of findings across response measures will likely lead to a broader understanding of the appropriate steps to take to make HMD-based VEs more useful in research and applications that involve spatial perception and cognition. This approach should include multiple measures of distance perception, as shown here, as well as further tests of spatial perception and cognition, such as judgments of perceived size and perceived potential for action.

\section{AUTHOR NOTE}

This work was supported by National Science Foundation Grant IIS0121084. We thank Scott Kuhl for technical assistance with the HMD. Correspondence concerning this article should be addressed to B. R. Kunz, Department of Psychology, University of Utah, 380 S. 1530 E., Room 502, Salt Lake City, UT 84112 (e-mail: benjamin.kunz@psych.utah.edu).

\section{REFERENCES}

Aglioti, S., DeSouza, J. F. X., \& Goodale, M. A. (1995). Size-contrast illusions deceive the eye but not the hand. Current Biology, 5, 679-685. ANDRE, J., \& Rogers, S. (2006). Using verbal and blind-walking dis- tance estimates to investigate the two visual systems hypothesis. Perception \& Psychophysics, 68, 353-361.

Bridgeman, B., Lewis, S., Heit, G., \& Nagle, M. (1979). Relation between cognitive and motor-oriented systems of visual position perception. Journal of Experimental Psychology: Human Perception \& Performance, 5, 692-700.

CAREY, D. P. (2001). Do action systems resist visual illusions? Trends in Cognitive Sciences, 5, 109-113.

Creem-Regehr, S. H., Willemsen, P., Gooch, A. A., \& Thompson, W. B. (2005). The influence of restricted viewing conditions on egocentric distance perception: Implications for real and virtual indoor environments. Perception, 34, 191-204.

Cutting, J. E., \& Vishton, P. M. (1995). Perceiving layout and knowing distances: The integration, relative potency, and contextual use of different information about depth. In W. Epstein \& S. J. Rogers (Eds.), Perception of space and motion (pp. 69-117). San Diego: Academic Press.

Durgin, F. H., Fox, L. F., Lewis, J., \& Walley, K. A. (2002, November). Perceptuomotor adaptation: More than meets the eye. Paper presented at the 43rd Annual Meeting of the Psychonomic Society, Kansas City, MO.

Fox, L. F., \& Durgin, F. H. (2003). More recalibration of the perception of linear self-motion [Abstract]. Journal of Vision, 3, 557a.

Franz, V. H. (2001). Action does not resist visual illusions. Trends in Cognitive Sciences, 5, 457-459.

Fukusima, S. S., Loomis, J. M., \& Da Silva, J. A. (1997). Visual perception of egocentric distance as assessed by triangulation. Journal of Experimental Psychology: Human Perception \& Performance, 23, 86-100

GILINSKY, A. S. (1951). Perceived size and distance in visual space. Psychological Review, 58, 460-482.

He, Z. J., Wu, B., OoI, T. L., Yarbrough, G., \& Wu, J. (2004). Judging egocentric distance on the ground: Occlusion and surface integration. Perception, 33, 789-806.

Hutchison, J. J., \& Loomis, J. M. (2006). Does energy expenditure affect the perception of egocentric distance? A failure to replicate Experiment 1 of Proffitt, Stefanucci, Banton, and Epstein (2003). Spanish Journal of Psychology, 9, 332-339.

Kelly, J. W., Loomis, J. M., \& BeAll, A. C. (2004). Judgments of exocentric direction in large-scale space. Perception, 33, 443-454.

Kuhl, S. A., Thompson, W. B., \& Creem-Regehr, S. H. (2008, August). HMD calibration and its effects on distance judgments. Paper presented at the ACM/SIGGRAPH Symposium on Applied Perception in Graphics and Visualization, Los Angeles.

Lappin, J. S., Shelton, A. L., \& Rieser, J. J. (2006). Environmental context influences visually perceived distance. Perception \& Psychophysics, 68, 571-581.

Loomis, J. M., Da Silva, J. A., Fujita, N., \& Fukusima, S. S. (1992). Visual space perception and visually directed action. Journal of Experimental Psychology: Human Perception \& Performance, 18, 906-921.

LOOMIS, J. M., \& KNAPP, J. M. (2003). Visual perception of egocentric distance in real and virtual environments. In L. J. Hettinger \& M. W. Haas (Eds.), Virtual and adaptive environments: Applications, implications, and human performance issues (pp. 21-46). Mahwah, NJ: Erlbaum.

LoOmis, J. M., \& PhILвECK, J. W. (2008). Measuring spatial perception with spatial updating and action. In R. L. Klatzky, B. MacWhinney, \& M. Behrmann (Eds.), Embodiment, ego-space, and action (pp. 1-43). New York: Taylor \& Francis, Psychology Press.

Messing, R., \& Durgin, F. H. (2005). Distance perception and the visual horizon in head-mounted displays. ACM Transactions on Applied Perception, 2, 234-250.

Milner, A. D., \& Goodale, M. A. (1995). The visual brain in action. Oxford: Oxford University Press.

Milner, A. D., \& Goodale, M. A. (2008). Two visual systems re-viewed. Neuropsychologia, 46, 774-785.

Mohler, B. J., Creem-Regehr, S. H., \& Thompson, W. B. (2006). The influence of feedback on egocentric distance judgments in real and virtual environments. In Proceedings of the Third SIGGRAPH Symposium on Applied Perception in Graphics and Visualization (pp. 9-14). New York: ACM Press.

Mohler, B. J., Thompson, W. B., Creem-Regehr, S. H., Willemsen, P., PICK, H. L., JR., \& RIESER, J. J. (2007). Calibration of locomotion result- 
ing from visual motion in a treadmill-based virtual environment. $A C M$ Transactions on Applied Perception, 4(1).

OoI, T. L., Wu, B., \& He, Z. J. (2001). Distance determined by the angular declination below the horizon. Nature, 414, 197-200.

OoI, T. L., Wu, B., \& He, Z. J. (2006). Perceptual space in the dark affected by the intrinsic bias of the visual system. Perception, $\mathbf{3 5}$, 605-624.

Philbeck, J. W., Behrmann, M., Black, S. E., \& Ebert, P. (2000). Intact spatial updating during locomotion after right posterior parietal lesions. Neuropsychologia, 38, 950-963.

Philbeck, J. W., Behrmann, M., Levy, L., Potolicchio, S. J., \& CAputy, A. J. (2004). Path integration deficits during linear locomotion after human medial temporal lobectomy. Journal of Cognitive Neuroscience, 16, 510-520.

Philbeck, J. W., \& Loomis, J. M. (1997). Comparison of two indicators of perceived egocentric distance under full-cue and reduced-cue conditions. Journal of Experimental Psychology: Human Perception \& Performance, 23, 72-85.

Philbeck, J. W., Loomis, J. M., \& Beall, A. C. (1997). Visually perceived location is an invariant in the control of action. Perception \& Psychophysics, 59, 601-612.

RichardSON, A. R., \& WALler, D. (2005). The effect of feedback training on distance estimation in virtual environments. Applied Cognitive Psychology, 19, 1089-1108.

RICHARDSON, A. R., \& WALLER, D. (2007). Interaction with an immersive virtual environment corrects users' distance estimates. Human Factors, 49, 507-517.

Rieser, J. J., Ashmead, D. H., Talor, C. R., \& Youngquist, G. A. (1990). Visual perception and the guidance of locomotion without vision to previously seen targets. Perception, 19, 675-689.

Sahm, C. S., Creem-Regehr, S. H., Thompson, W. B., \& WillemSEN, P. (2005). Throwing versus walking as indicators of distance perception in similar real and virtual environments. ACM Transactions on Applied Perception, 2, 35-45.

SedGwick, H. A. (1986). Space perception. In K. R. Boff, L. Kaufman, \& J. P. Thomas (Eds.), Handbook of perception and performance (Vol. 1, pp. 21-1 to 21-57). New York: Wiley.

SinAI, M. J., OoI, T. L., \& He, Z. J. (1998). Terrain influences the accurate judgement of distance. Nature, 395, 497-500.
Thompson, W. B., Dilda, V., \& Creem-Regehr, S. H. (2007). Absolute distance perception to locations off the ground plane. Perception, 36, 1559-1571.

Thompson, W. B., Willemsen, P., Gooch, A. A., Creem-Regehr, S. H., Loomis, J. M., \& Beall, A. C. (2004). Does the quality of the computer graphics matter when judging distance in visually immersive environments? Presence: Teleoperators \& Virtual Environments, 13, 560-571.

Waller, D., \& Richardson, A. R. (2008). Correcting distance estimates by interacting with immersive virtual environments: Effects of task and available sensory information. Journal of Experimental Psychology: Applied, 14, 61-72.

Willemsen, P., Colton, M. B., Creem-Regehr, S. H., \& Thompson, W. B. (2009). The effects of head-mounted display mechanical properties and field-of-view on distance judgments in virtual environments. ACM Transactions on Applied Perception, 6(2).

Willemsen, P., \& Gooch, A. A. (2002). Perceived egocentric distances in real, image-based, and traditional virtual environments. In Proceedings of IEEE Virtual Reality Conference 2002 (p. 275). Washington, DC: IEEE Computer Society.

Willemsen, P., Gooch, A. A., Thompson, W. B., \& Creem-Regehr, S. H. (2008). Effects of stereo viewing conditions on distance perception in virtual environments. Presence: Teleoperators \& Virtual Environments, 17, 91-101.

WitMer, B. G., \& SADOWSKI, W. J., JR. (1998). Nonvisually guided locomotion to a previously viewed target in real and virtual environments. Human Factors, 40, 478-488.

Witt, J. K., Stefanucci, J. K., Riener, C. R., \& Proffitt, D. R. (2007). Seeing beyond the target: Environmental context affects distance perception. Perception, 36, 1752-1768.

Wolbers, T., Hegarty, M., Büchel, C., \& Loomis, J. M. (2008). Spatial updating: How the brain keeps track of changing object locations during observer motion. Nature Neuroscience, 11, 1223-1230.

Wu, B., OoI, T. L., \& He, Z. J. (2004). Perceiving distance accurately by a directional process of integrating ground information. Nature, 428, $73-77$.

(Manuscript received December 24, 2008; revision accepted for publication March 9, 2009.) 